\title{
Existence of nonnegative solutions for a nonlinear fractional boundary value problem
}

\author{
Assia Guezane-Lakoud and Kheireddine Belakroum \\ Communicated by Evren Hincal
}

\begin{abstract}
This paper deals with the existence of solutions for a class of boundary value problem (BVP) of fractional differential equation with three point conditions via LeraySchauder nonlinear alternative. Moreover, the existence of nonnegative solutions is discussed.
\end{abstract}

Keywords. Positive solution, Caputo fractional derivative, fixed point theorems.

2010 Mathematics Subject Classification. 34B18, 26 A33.

\section{Introduction}

Fractional differential equations are a natural generalization of ordinary differential equations. They can describe many phenomena in various fields of science and engineering such as control, porous media, electrochemistry, etc. It has been established that, in many situations these models provide more suitable results than analogous models with integer derivatives. As a consequence, the subject of fractional differential equations is gaining much importance and attention. For details, see $[1-6,8]$ and references therein.

It should be noted that most of the papers and books on fractional calculus are devoted to the solvability of linear initial value fractional differential equation in terms of special functions $[20,25]$. Recently, there are some papers dealing with the existence and multiplicity of solutions or nonnegative solutions of nonlinear initial value fractional differential equation by the use of techniques of nonlinear analysis of such fixed point theorems.

For example, Zhoujin [9] considered the fractional differential equation

$$
\begin{aligned}
& { }^{c} D^{\alpha} u(t)+f\left(t, u(t),{ }^{c} D_{a^{+}}^{\beta} u(t)\right)=0, \quad 0<t<1, \quad 3<\alpha \leq 4, \\
& u(0)=u^{\prime}(0)=u^{\prime \prime}(0)=0, \quad u(1)=u(\xi), \quad 0<\xi<1,
\end{aligned}
$$

where ${ }^{c} D^{\alpha}$ denotes the Caputo's fractional derivative, $\beta>0, \alpha-\beta \geq 1$. The existence results are derived by means of Schauder's fixed-point theorem. 
Liang and Zhang [22] studied the existence and uniqueness of positive solutions by properties of the Green function, the lower and upper solution method and fixed point theorem for the fractional boundary value problem

$$
\begin{aligned}
& D_{0^{+}}^{q} u(t)+f(t, u(t))=0, \quad 0<t<1, \\
& u(0)=u^{\prime}(0)=0, \quad u^{\prime}(1)=\sum_{i=1}^{m-2} \beta_{i} u^{\prime}\left(\zeta_{i}\right),
\end{aligned}
$$

where $2<q \leq 3$ and $D_{0^{+}}^{q}$ is the Riemann-Liouville fractional derivative.

In [27], Mujeeb studied existence and multiplicity results by means of the GuoKrasnosel'skii fixed point theorem, for a coupled system of nonlinear nonlocal boundary value problems for higher order fractional differential equations of the type

$$
\begin{gathered}
{ }^{c} D_{0^{+}}^{q} u(t)=\lambda a(t) f(u(t), v(t)), \quad{ }^{c} D_{0^{+}}^{q} u(t)=\mu b(t) g(u(t), v(t)), \\
u^{\prime}(0)=u^{\prime \prime}(0)=u^{\prime \prime \prime}(0)=\ldots=u^{(n-1)}(0)=0, \quad u(1)=\xi_{1} u\left(\eta_{1}\right), \\
v^{\prime}(0)=v^{\prime \prime}(0)=v^{\prime \prime \prime}(0)=\ldots=v^{(n-1)}(0)=0, \quad v(1)=\xi_{2} u\left(\eta_{2}\right),
\end{gathered}
$$

where $\lambda, \mu>0, n-1<\alpha, \beta \leq n$ for $n \in \mathbb{N} ; \xi_{i}, \eta_{i} \in(0,1)$ for $i=1,2$ and $D_{0^{+}}^{q}$ is the Caputo's fractional derivative.

Ahmad et al. [2] studied the nonlinear fractional differential equation with nonlocal boundary value

$$
\begin{aligned}
& { }^{c} D_{0^{+}}^{q} u(t)=f(t, u(t)), \quad 0<t<1, \\
& x(0)=x^{\prime}(0)=x^{\prime \prime}(0)=\ldots=x^{(m-2)}(0)=0, \quad x(1)=a x(\eta),
\end{aligned}
$$

in which $q \in(m-1, m], m \in \mathbb{N}, m \geq 2$. Existence results are based on the contraction mapping principle and Krasnoselskii's fixed-point theorem.

Motived by all of the works above, in this work, we consider the existence and uniqueness of nonnegative solutions of boundary value problem for nonlinear fractional differential equation

$$
\begin{aligned}
& { }^{c} D_{a^{+}}^{q} u(t)=f\left(t, u(t),{ }^{c} D_{a^{+}}^{\sigma} u(t)\right), \quad 0<t<1, \\
& u(0)=u^{\prime \prime}(0)=0, u^{\prime}(\eta)=\alpha u^{\prime \prime}(1),
\end{aligned}
$$

where $f:[0,1] \times \mathbb{R} \times \mathbb{R} \rightarrow \mathbb{R}$ is a given function, $2<q<3,0<\sigma<1$, $0<\eta<1$ and ${ }^{c} D_{a^{+}}^{q}$ denotes the Caputo's fractional derivative.

We remark that the Caputo fractional derivative is more suitable than the usual Riemann-Liouville derivative for the applications in several engineering problems 
due to the fact that it has better relations with the Laplace transform and because the differentiation appears inside instead of outside of the integral, so to alleviate the effects of noise and numerical differentiation (see $[23,29,30])$. The rest of paper is organized as follows. In Section 2, we cite some definitions and lemmas needed in our proofs. Section 3 treats the existence and uniqueness of solution by using Banach contraction principle, Leray Schauder nonlinear alternative. Section 4 is devoted to prove the existence of nonnegative solutions with the help of Guo-Krasnoselskii theorem. Last, we give some examples illustrating the previous results.

\section{Preliminaries and Lemmas}

In this section we present some lemmas and definitions from fractional calculus theory, see [22], which will be needed throughout the paper.

Definition 2.1. If $g \in C[0,1]$ and $0<\alpha$, then the Riemann-Liouville fractional integral is defined by

$$
I_{a^{+}}^{\alpha} g(t)=\frac{1}{\Gamma(\alpha)} \int_{\alpha}^{t} \frac{g(s)}{(t-s)^{1-\alpha}} d s .
$$

Let $\alpha \geq 0, n=[\alpha]+1$. If $g \in C^{n}[a, b]$ then the Caputo fractional derivative of order $\alpha$ of $g$ defined by

$$
{ }^{c} D_{a^{+}}^{\alpha} g(t)=\frac{1}{\Gamma(n-\alpha)} \int_{\alpha}^{t} \frac{g^{(n)}(s)}{(t-s)^{\alpha-n+1}} d s
$$

exists almost every where on $[a, b]([\alpha]$ is the entire part of $\alpha)$.

Lemma 2.2. Let $\alpha, \beta \geq 0$ and $n=[\alpha]+1$, then the following relation holds:

$$
{ }^{c} D_{0^{+}}^{\alpha} t^{\beta-1}=\frac{\Gamma(\beta) t^{\beta-\alpha-1}{ }^{c}}{\Gamma(\beta-\alpha)} D_{0^{+}}^{\alpha} t^{k}=0, \quad k=0,1,2, \ldots, n-1 .
$$

Lemma 2.3. [18] For $\alpha \geq 0$ and $g(t) \in C[0,1]$, the homogeneous fractional differential equation

$$
{ }^{c} D_{a^{+}}^{\alpha} g(t)=0
$$

has a solution

$$
g(t)=c_{1}+c_{2} t+c_{3} t^{2}+\ldots+c_{n} t^{n-1},
$$

where $c_{i} \in \mathbb{R}, i=0, \ldots, n$ and $n=[\alpha]+1$. 
Denote by $L^{1}([0,1], \mathbb{R})$ the Banach space of Lebesgue integrable functions from $[0,1]$ into $\mathbb{R}$ with the norm $\|y\|=\int_{0}^{1}|y(t)| d t$.

The following lemmas give some properties of Riemann-Liouville fractional integrals and Caputo fractional derivative.

Lemma 2.4. Let $p, q \geq 0, f \in L_{1}[a, b]$. Then,

$$
I_{0^{+}}^{p} I_{0^{+}}^{q} f(t)=I_{0^{+}}^{p+q} f(t)=I_{0^{+}}^{q} I_{0^{+}}^{p} f(t)
$$

and

$$
{ }^{c} D_{0^{+}}^{q} I_{0^{+}}^{q} f(t)=f(t), \forall t \in[a, b] .
$$

Lemma 2.5. [23] Let $\beta>\alpha>0$. Then, the formula ${ }^{c} D_{0^{+}}^{\alpha} I_{0^{+}}^{\beta} f(t)=I_{0^{+}}^{\beta-\alpha} f(t)$ holds almost everywhere on $t \in[a, b]$ for $f \in L_{1}[a, b]$ and it is valid at any point $x \in[a, b]$ if $f \in C[a, b]$.

Now, we give solution of an auxiliary problem.

Lemma 2.6. Let $2<q<3,0<\sigma<1$ and $y \in C[a, b]$. The unique solution of the fractional boundary value problem

$$
\begin{cases}{ }^{c} D_{a^{+}}^{q} u(t)=y(t), & 0<t<1, \\ u(0)=u^{\prime \prime}(0)=0, & u^{\prime}(\eta)=\alpha u^{\prime \prime}(1)\end{cases}
$$

is given by

$$
u(t)=\frac{1}{\Gamma(q-2)}\left[\int_{0}^{1} G(t, s) y(s) d s-\frac{t}{q-2} \int_{0}^{\eta}(\eta-s)^{q-2} y(s) d s\right],
$$

where

$$
G(t, s)= \begin{cases}\frac{(t-s)^{q-1}}{(q-2)(q-1)}+\frac{t \alpha}{(1-s)^{3-q}}, & s \leq t \\ \frac{t \alpha}{(1-s)^{3-q}}, & s>t .\end{cases}
$$

Proof. Applying Lemmas 2.3 and 2.4 to (9), we get

$$
u(t)=I_{0^{+}}^{q} y(t)+c_{1}+c_{2} t+c_{3} t^{2} .
$$


Differentiating both sides of (12) and using Lemma 2.5 it yields

$$
\begin{aligned}
& u^{\prime}(t)=I_{0^{+}}^{q-1} y(t)+c_{2}+c_{3} t, \\
& u^{\prime \prime}(t)=I_{0^{+}}^{q-2} y(t)+c_{3} .
\end{aligned}
$$

The first condition in (9) implies $c_{1}=c_{3}=0$, the second one gives

$$
c_{2}=\alpha I_{0^{+}}^{q-2} y(1)-I_{0^{+}}^{q-1} y(\eta) .
$$

Substituting $c_{2}$ by its value in (12), we obtain

$$
u(t)=I_{0^{+}}^{q} y(t)+t\left(\alpha I_{0^{+}}^{q-2} y(1)-I_{0^{+}}^{q-1} y(\eta)\right),
$$

that can be written as

$$
\begin{aligned}
u(t) & =\frac{1}{\Gamma(q)} \int_{0}^{t} \frac{y(s)}{(t-s)^{1-q}} d s+\frac{t \alpha}{\Gamma(q-2)} \int_{0}^{1} \frac{y(s)}{(1-s)^{3-q}} d s \\
& -\frac{t}{\Gamma(q-1)} \int_{0}^{\eta} \frac{y(s)}{(\eta-s)^{(2-q)}} d s,
\end{aligned}
$$

i.e.,

$$
u(t)=\frac{1}{\Gamma(q-2)}\left[\int_{0}^{1} G(t, s) y(s) d s-\frac{t}{q-2} \int_{0}^{\eta}(\eta-s)^{q-2} y(s) d s\right],
$$

where $G$ is defined by (11). The proof is complete.

\section{Existence and Uniqueness Results}

In this section we prove the existence and uniqueness of solutions in the Banach space $\mathrm{E}$ of all functions $u \in C[0,1]$ into $\mathbb{R}$ with the norm

$$
\|u\|=\max _{0 \leq t \leq 1}|u|+\max _{0 \leq t \leq 1}\left|{ }^{c} D_{0^{+}}^{\sigma} u\right| .
$$

We know that ${ }^{c} D_{0^{+}}^{\sigma} u \in C[0,1]$ if $0<\sigma<1$.

Denote by $E^{+}=\{u \in E, u(t) \geq 0, t \in[0,1]\}$. Throughout this section, we suppose that $f \in C([0,1] \times \mathbb{R} \times \mathbb{R}, \mathbb{R})$. We define the integral operator $T: E \rightarrow E$ by

$$
\begin{gathered}
T u(t)=\frac{1}{\Gamma(q-2)}\left[\int_{0}^{1} G(t, s) f\left(s, u(s),{ }^{c} D_{0^{+}}^{\sigma} u(s)\right) d s\right. \\
\left.-\frac{t}{q-2} \int_{0}^{\eta}(\eta-s)^{q-2} f\left(s, u(s),{ }^{c} D_{0^{+}}^{\sigma} u(s)\right) d s\right] .
\end{gathered}
$$


Then, we have the following lemma.

Lemma 3.1. The function $u \in E$ is solution of the fractional $B V P(1)$ if and only if $T u(t)=u(t)$ for all $t \in[0,1]$.

Theorem 3.2. Assume that there exist nonnegative functions $h, g \in L^{1}\left([0,1], \mathbb{R}_{+}\right)$ such that for all $x, y \in \mathbb{R}$ and $t \in[0,1]$, one has

$$
\begin{aligned}
& |f(t, x, \bar{x})-f(t, y, \bar{y})| \leq g(t)|x-y|+h(t)|\bar{x}-\bar{y}|, \\
& C_{g}+C_{h}<1 \text { and } A_{g}+A_{h}<(1-\sigma) \Gamma(1-\sigma),
\end{aligned}
$$

where

$$
\begin{aligned}
& C_{g}=\left\|I_{0^{+}}^{q-1} g\right\|_{L^{1}}+|\alpha| I_{0^{+}}^{q-2} g(1)+I_{0^{+}}^{q-1} g(\eta), \\
& C_{h}=\left\|I_{0^{+}}^{q-1} h\right\|_{L^{1}}+|\alpha| I_{0^{+}}^{q-2} h(1)+I_{0^{+}}^{q-1} h(\eta)
\end{aligned}
$$

and

$$
\begin{aligned}
& A_{g}=I_{0^{+}}^{q-1}(g(1)+g(\eta))++|\alpha| I_{0^{+}}^{q-2} g(1), \\
& A_{h}=I_{0^{+}}^{q-1}(h(1)+h(\eta))++|\alpha| I_{0^{+}}^{q-2} h(1) .
\end{aligned}
$$

Then, the fractional BVP (1) has unique solution $u \in E$.

To prove Theorem 3.2, we use the following property of Riemann-Liouville fractional integrals.

Lemma 3.3. [12] Let $q>0, f \in L_{1}\left([a, b], \mathbb{R}_{+}\right)$. Then, for all $t \in[a, b]$ we have

$$
I_{0^{+}}^{q+1} f(t) \leq\left\|I_{0^{+}}^{q} f\right\|_{L^{1}} .
$$

Now we prove Theorem 3.2.

Proof of Theorem 3.2. We transform the fractional boundary value problem to a fixed point problem. By Lemma 3.1, the fractional boundary value problem (1) has a solution if and only if the operator $T$ has a fixed point in $E$. Now we will prove that $T$ is a contraction. Let $u, v \in E$. Then,

$$
\begin{gathered}
T u(t)-T v(t)=\frac{1}{\Gamma(q-2)} \\
\times \int_{0}^{1} G(t, s)\left(f\left(s, u(s),{ }^{c} D_{0^{+}}^{\sigma} u(s)\right)-f\left(s, v(s),{ }^{c} D_{0^{+}}^{\sigma} v(s)\right)\right) d s
\end{gathered}
$$




$$
\begin{aligned}
& -\frac{t}{(q-2)} \int_{0}^{\eta}(\eta-s)^{q-2}\left(f\left(s, u(s),{ }^{c} D_{0^{+}}^{\sigma} u(s)\right)-f\left(s, v(s),{ }^{c} D_{0^{+}}^{\sigma} v(s)\right)\right) d s \\
& \quad=I_{0^{+}}^{q}\left(\left(f\left(t, u(t),{ }^{c} D_{0^{+}}^{\sigma} u(t)\right)-f\left(t, v(t),{ }^{c} D_{0^{+}}^{\sigma} v(t)\right)\right)\right) \\
& +t \alpha I_{0^{+}}^{q-2}\left(f\left(1, u(1),{ }^{c} D_{0^{+}}^{\sigma} u(1)\right)-f\left(1, v(1),{ }^{c} D_{0^{+}}^{\sigma} v(1)\right)\right) \\
& -t I_{0^{+}}^{q-2}\left(f\left(\eta, u(\eta),{ }^{c} D_{0^{+}}^{\sigma} u(\eta)\right)-f\left(\eta, v(\eta),{ }^{c} D_{0^{+}}^{\sigma} v(\eta)\right)\right) .
\end{aligned}
$$

With the help of (18), we obtain

$$
\begin{gathered}
|T u(t)-T v(t)| \\
\leq \max _{0 \leq t \leq 1}|u(t)-v(t)|\left[I_{0^{+}}^{q} g(t)+|\alpha| I_{0^{+}}^{q-2} g(1)+I_{0^{+}}^{q-1} g(\eta)\right] \\
+\max _{0 \leq t \leq 1}\left|{ }^{c} D_{0^{+}}^{q} u(t)-{ }^{c} D_{0^{+}}^{q} v(t)\right|\left[I_{0^{+}}^{q} h(t)+|\alpha| I_{0^{+}}^{q-2} h(1)+I_{0^{+}}^{q-1} h(\eta)\right] .
\end{gathered}
$$

Lemma 3.3 implies

$$
\begin{aligned}
|T u(t)-T v(t)| & \leq\|u-v\|\left\{\left\|I_{0^{+}}^{q-1} g\right\|+|\alpha| I_{0^{+}}^{q-2} g(1)+I_{0^{+}}^{q-1} g(\eta)\right. \\
& \left.+\left\|I_{0^{+}}^{q-1} h\right\|+|\alpha| I_{0^{+}}^{q-2} h(1)+I_{0^{+}}^{q-1} h(\eta)\right\} \\
& \leq\|u-v\|\left(C_{g}+C_{h}\right) .
\end{aligned}
$$

In view of (18) it yields

$$
|T u(t)-T v(t)| \leq\|u-v\| .
$$

On other hand we have

$$
{ }^{c} D_{0^{+}}^{\sigma} T u(t)-{ }^{c} D_{0^{+}}^{\sigma} T v(t)=\frac{1}{\Gamma(1-\sigma)} \int_{0}^{t} \frac{(T u)^{\prime}(s)-(T v)^{\prime}(s)}{(t-s)^{\sigma}} d s,
$$

where

$$
\begin{aligned}
(T u)^{\prime}(t)= & \frac{1}{\Gamma(q-2)}\left[\int_{0}^{1} G_{1}(t, s) f\left(s, u(s),{ }^{c} D_{0^{+}}^{\sigma} u(s)\right) d s\right. \\
& \left.-\frac{1}{(q-2)} \int_{0}^{\eta}(\eta-s)^{q-2} f\left(s, u(s),{ }^{c} D_{0^{+}}^{\sigma} u(s)\right) d s\right]
\end{aligned}
$$




$$
G_{1}(t, s)=\frac{\partial G(t, s)}{\partial t}= \begin{cases}\frac{(t-s)^{q-2}}{(q-2)}+\frac{\alpha}{(1-s)^{3-q}} & s \leq t \\ \frac{\alpha}{(1-s)^{3-q}} & t \leq s .\end{cases}
$$

Therefore,

$$
\begin{aligned}
& { }^{c} D_{0^{+}}^{\sigma} T u(t)-{ }^{c} D_{0^{+}}^{\sigma} T v(t)=\frac{1}{\Gamma(q-2) \Gamma(1-\sigma)} \\
& \times\left[\int _ { 0 } ^ { t } ( t - s ) ^ { - \sigma } \left(\int_{0}^{1} G_{1}(s, r)\right.\right. \\
& \times\left(f\left(r, u(r),{ }^{c} D_{0^{+}}^{\sigma} u(r)-f\left(r, v(r),{ }^{c} D_{0^{+}}^{\sigma} v(r)\right)\right) d r\right. \\
& -\frac{1}{\Gamma(q-2)} \int_{0}^{\eta}(\eta-r)^{q-2} \\
& \left.\left.\times\left(f\left(r, u(r),{ }^{c} D_{0^{+}}^{\sigma} u(r)\right)-f\left(r, v(r),{ }^{c} D_{0^{+}}^{\sigma} v(r)\right)\right) d r\right) d s\right] .
\end{aligned}
$$

Applying (18) we get

$$
\begin{gathered}
\left|{ }^{c} D_{0^{+}}^{\sigma} T u(t)-{ }^{c} D_{0^{+}}^{\sigma} T v(t)\right| \leq \frac{1}{\Gamma(q-2) \Gamma(1-\sigma)} \\
\times\left[( t - s ) ^ { - \sigma } \int _ { 0 } ^ { t } \left(\max _{0 \leq t \leq 1}|u-v|\right.\right. \\
\times\left(\int_{0}^{1} G_{1}(s, r) g(r) d r-\int_{0}^{\eta} \frac{(\eta-r)^{q-2}}{(q-2)} g(r) d r\right) \\
\times\left(\left.\int_{0 \leq t \leq 1}\right|^{c} D_{0^{+}}^{\sigma} u-{ }^{c} D_{0^{+}}^{\sigma} v \mid\right. \\
\left.\left.\quad G_{1}(s, r) h(r) d r-\int_{0}^{\eta} \frac{(\eta-r)^{q-2}}{(q-2)} h(r) d r\right)\right] d s .
\end{gathered}
$$

Let us estimate the term

$$
\int_{0}^{1} G_{1}(s, r) g(r) d r-\int_{0}^{\eta} \frac{(\eta-r)^{q-2}}{(q-2)} g(r) d r .
$$


We have

$$
\begin{aligned}
& \left|\int_{0}^{1} G(s, r) g(r) d r-\frac{s}{(q-2)} \int_{0}^{\eta} \frac{g(r)}{(\eta-r)^{2-q}} d r\right| \\
& =\left|\Gamma(q-2)\left[I_{0^{+}}^{q} g(s)+s\left(\alpha I_{0^{+}}^{q-2} g(1)-I_{0^{+}}^{q-1} g(\eta)\right)\right]\right| \\
& \left|\int_{0}^{1} G_{1}(s, r) g(r) d r-\int_{0}^{\eta} \frac{(\eta-r)^{q-2}}{(q-2)} g(r) d r\right| \\
& =\left|\Gamma(q-2)\left[I_{0^{+}}^{q-1} g(s)+\alpha I_{0^{+}}^{q-2} g(1)-I_{0^{+}}^{q-1} g(\eta)\right]\right| \\
& \leq \Gamma(q-2)\left[I_{0^{+}}^{q-1} g(s)+\alpha I_{0^{+}}^{q-2} g(1)+I_{0^{+}}^{q-1} g(\eta)\right] \\
& \leq \Gamma(q-2) A_{g}
\end{aligned}
$$

and consequently (29) becomes

$$
\left|{ }^{c} D_{0^{+}}^{\sigma} T u(t)-{ }^{c} D_{0^{+}}^{\sigma} T v(t)\right|=\frac{\|u-v\|}{(1-\sigma) \Gamma(1-\sigma)}\left(A_{g}+A_{h}\right) .
$$

With the help of (22) it yields

$$
\left|{ }^{c} D_{0^{+}}^{\sigma} T u(t)-{ }^{c} D_{0^{+}}^{\sigma} T v(t)\right| \leq\|u-v\| .
$$

Taking into account (25)-(32), we obtain

$$
\|T u-T v\| \leq\|u-v\| .
$$

From here, the contraction principle ensures the uniqueness of solution for the fractional boundary value problem (1), this finishes the proof.

Now, we give an existence result for the fractional boundary value problem (1).

Theorem 3.4. Assume that $f(t, 0,0) \neq 0$ and there exists nonnegative functions $k, h, g \in L^{1}\left([0,1], \mathbb{R}_{+}\right), \phi, \psi \in C\left(\mathbb{R}_{+}, \mathbb{R}_{+}^{*}\right)$ are nondecreasing on $\mathbb{R}_{+}$and $r>0$ such that

$$
\begin{gathered}
|f(t, x, \bar{x})| \leq k(t) \psi(|x|)+h(t) \phi(|x|)+g(t), \\
\text { a.e. }(t, x) \in[0,1] \times \mathbb{R}^{2}, \\
(\psi(r)+\phi(r)+1)\left(C_{1}+\frac{C_{2}}{(1-\sigma) \Gamma(1-\sigma)}\right)<r,
\end{gathered}
$$

where

$$
C_{1}=\max _{0 \leq t \leq 1}\left\{C_{k}, C_{h}, C_{g}\right\}, C_{2}=\max _{0 \leq t \leq 1}\left\{A_{k}, A_{h}, A_{g}\right\},
$$


$C_{h, g}, A_{h, g}$ are defined as in Theorem 3.2 and

$$
\begin{aligned}
& A_{k}=I_{0^{+}}^{q-1}(k(1)+k(\eta))++|\alpha| I_{0^{+}}^{q-2} k(1), \\
& C_{k}=\left\|I_{0^{+}}^{q-1} k\right\|_{L^{1}}+|\alpha| I_{0^{+}}^{q-2} k(1)+I_{0^{+}}^{q-1} k(\eta) .
\end{aligned}
$$

Then, the fractional boundary value problem (1) has at least one nontrivial solution $u^{*}$ in $E$.

To prove this theorem, we apply Leray-Schauder nonlinear alternative.

Lemma 3.5. [10] Let $F$ be a Banach space and $\Omega$ a bounded open subset of $F \in$ $\Omega, \quad T: \bar{\Omega} \rightarrow F$ be a completely continuous operator. Then, either there exists $x \in \partial \Omega, \lambda>1$ such that $T(x)=\lambda x$, or there exists a fixed point $x^{*} \in \bar{\Omega}$.

Proof of Theorem 3.4. First, let us prove that $T$ is completely continuous. It is clear that $T$ is continuous since $f$ and $G$ are continuous. Let $B_{r}=\{u \in E,\|u\| \leq$ $r$ \} be a bounded subset in $E$. We shall prove that $T\left(B_{r}\right)$ is relatively compact.

(i) For $u \in B_{r}$ and using (34) we get

$$
\begin{aligned}
& |T u(t)| \leq \frac{1}{\Gamma(q-2)} \\
& \times \int_{0}^{1}|G(t, s)|\left[k(s) \psi(|u(s)|)+h(s) \phi\left(\left|{ }^{c} D_{0^{+}}^{\sigma} u(s)\right|\right)+g(s)\right] d s \\
& +\frac{t}{\Gamma(q-2)} \int_{0}^{\eta}(\eta-s)^{q-2}\left[k(s) \psi(|u(s)|)+h(s) \phi\left(\left|{ }^{c} D_{0^{+}}^{\sigma} u(s)\right|\right)\right] .
\end{aligned}
$$

Since $\phi$ and $\psi$ are nondecreasing (36) implies

$$
\begin{aligned}
|T u(t)| & \leq \frac{1}{\Gamma(q-2)} \int_{0}^{1}|G(t, s)|[k(s) \psi(\|u\|)+h(s) \phi(\|u\|)+g(s)] d s \\
& +\frac{t}{\Gamma(q-2)} \int_{0}^{\eta}(\eta-s)^{q-2}[k(s) \psi(\|u\|)+h(s) \phi(\|u\|)] \\
& \leq \frac{1}{\Gamma(q-2)} \int_{0}^{1}|G(t, s)|[k(s) \psi(r)+h(s) \phi(r)+g(s)] d s \\
& +\frac{t}{\Gamma(q-2)} \int_{0}^{\eta}(\eta-s)^{q-2}[k(s) \psi(r)+h(s) \phi(r)] d s .
\end{aligned}
$$


Using similar techniques to get (25) it yields

$$
\begin{aligned}
|T u(t)| & \leq \psi(r)\left[\left\|I_{0^{+}}^{q-1} k\right\|_{L^{1}}+|\alpha| I_{0^{+}}^{q-2} k(1)+I_{0^{+}}^{q-1} k(\eta)\right] \\
& +\phi(r)\left[\left\|I_{0^{+}}^{q-1} h\right\|_{L^{1}}+|\alpha| I_{0^{+}}^{q-2} h(1)+I_{0^{+}}^{q-1} h(\eta)\right] \\
& +\left\|I_{0^{+}}^{q-1} g\right\|_{L^{1}}+|\alpha| I_{0^{+}}^{q-2} g(1)+I_{0^{+}}^{q-1} g(\eta) \\
& \leq C_{k} \psi(r)+C_{h} \phi(r)+C_{g} .
\end{aligned}
$$

Hence,

$$
|T u(t)| \leq C_{1}(\psi(r)+\phi(r)+1) .
$$

Moreover, we have

$$
\begin{aligned}
&\left|(T u)^{\prime}(t)\right|=\mid \frac{1}{\Gamma(q-2)}\left[\int_{0}^{1} G_{1}(t, s) f\left(s, u(s),{ }^{c} D_{0^{+}}^{\sigma} u(s)\right) d s\right. \\
&\left.-\frac{1}{(q-2)} \int_{0}^{\eta}(\eta-s)^{q-2} f\left(s, u(s),{ }^{c} D_{0^{+}}^{\sigma} u(s)\right) d s\right] \mid \\
& \leq \frac{1}{\Gamma(q-2)} \\
& \times \mid \int_{0}^{1} G_{1}(t, s)[k(s) \psi(r)+h(s) \phi(r)+g(s)] d s \\
&-\frac{1}{(q-2)} \int_{0}^{\eta}(\eta-s)^{q-2}[k(s) \psi(r)+h(s) \phi(r)] d s \mid \\
& \leq \frac{1}{\Gamma(q-2)}\left[\mid \psi(r) \int_{0}^{1} G_{1}(t, s) k(s) d s\right. \\
&\left.-\frac{1}{(q-2)} \int_{0}^{\eta}(\eta-s)^{q-2} k(s) d s \mid\right] \\
&+\left[|| \begin{array}{l}
\phi \\
\phi
\end{array} \int_{0}^{1} G_{1}(t, s) h(s) d s-\frac{1}{(q-2)} \int_{0}^{\eta}(\eta-s)^{q-2} h(s) d s \mid\right] \\
&\left.+|| \int_{0}^{1} G_{1}(t, s) g(s) d s-\frac{1}{(q-2)} \int_{0}^{\eta}(\eta-s)^{q-2} g(s) d s \mid\right], \\
&\left|(T u)^{\prime}(t)\right| \leq A_{k} \psi(r)+A_{h} \phi(r)+A_{g}, \\
&\left|(T u)^{\prime}(t)\right| \leq C_{2}(\psi(r)+\phi(r)+1) .
\end{aligned}
$$


Using (30) we obtain

$$
\begin{aligned}
\left|{ }^{c} D_{0^{+}}^{q} T u(t)\right| & \leq \frac{1}{\Gamma(1-\sigma)} \int_{0}^{t} \frac{A_{k} \psi(r)+A_{h} \phi(r)+A_{g}}{(t-s)^{\sigma}} d s \\
& \leq \frac{C_{2}}{\Gamma(1-\sigma)} \int_{0}^{t} \frac{\psi(r)+\phi(r)+1}{(t-s)^{\sigma}} d s \\
& \leq \frac{C_{2}}{(1-\sigma) \Gamma(1-\sigma)}(\psi(r)+\phi(r)+1)
\end{aligned}
$$

and from (39) and (43), we get

$$
\|T u\|=((\psi(r)+\phi(r)+1))\left(C_{1}+\frac{C_{2}}{(1-\sigma) \Gamma(1-\sigma)}\right) .
$$

Then $T\left(B_{r}\right)$ is uniformly bounded.

(ii) $T\left(B_{r}\right)$ is equicontinuous indeed for all $t_{1}, t_{2} \in[0,1], t_{1}<t_{2}, u \in B_{r}$. Let

$$
C=\max _{0 \leq t \leq 1}\left\{\left|f\left(t, u(t),{ }^{c} D_{0^{+}}^{\sigma} u(t)\right)\right|, 0 \leq t \leq 1,\|u\|<r\right\} .
$$

Therefore,

$$
\begin{aligned}
\left|T u\left(t_{1}\right)-T u\left(t_{2}\right)\right| & =\int_{t_{1}}^{t_{2}}\left|(T u)^{\prime}(t)\right| d t \\
& \leq \int_{t_{1}}^{t_{2}}\left(A_{k} \psi(r)+A_{h} \phi(r)+A_{g}\right) d t \\
& \leq\left(t_{1}-t_{2}\right)\left(A_{k} \psi(r)+A_{h} \phi(r)+A_{g}\right) .
\end{aligned}
$$

On the other hand, we have

$$
\begin{aligned}
& \left|{ }^{c} D_{0^{+}}^{\sigma} T u\left(t_{1}\right)-{ }^{c} D_{0^{+}}^{\sigma} T u\left(t_{2}\right)\right| \\
& =\left|\frac{1}{\Gamma(1-\sigma)} \int_{0}^{t_{1}} \frac{(T u)^{\prime}(s)}{\left(t_{1}-s\right)^{\sigma}} d s-\frac{1}{\Gamma(1-\sigma)} \int_{0}^{t_{2}} \frac{(T u)^{\prime}(s)}{\left(t_{2}-s\right)^{\sigma}} d s\right| \\
& \leq \frac{1}{\Gamma(1-\sigma)} \int_{0}^{t_{1}}\left|\left(t_{1}-s\right)^{-\sigma}-\left(t_{2}-s\right)^{-\sigma}\right|\left|(T u)^{\prime}(s)\right| d s \\
& +\frac{1}{\Gamma(1-\sigma)} \int_{t_{1}}^{t_{2}}\left(t_{2}-s\right)^{-\sigma}\left|(T u)^{\prime}(s)\right| d s .
\end{aligned}
$$

Using (30) and (40) it yields

$$
\left|(T u)^{\prime}(t)\right| \leq C_{2}(\psi(r)+\phi(r)+1),
$$




$$
\begin{aligned}
& \left|{ }^{c} D_{0^{+}}^{q} T u\left(t_{1}\right)-{ }^{c} D_{0^{+}}^{q} T u\left(t_{2}\right)\right| \\
& \leq \frac{C_{2}(\psi(r)+\phi(r)+1)}{(1-\sigma) \Gamma(1-\sigma)}\left(2\left(t_{2}-t_{1}\right)^{1-\sigma}+t_{2}^{1-\sigma}+t_{1}^{1-\sigma}\right),
\end{aligned}
$$

when $t_{1} \rightarrow t_{2}$, in (45) and (48), $\mid T u\left(t_{1)}-T u\left(t_{2}\right) \mid\right.$ and $\left|{ }^{c} D_{0^{+}}^{q} T u\left(t_{1}\right)-{ }^{c} D_{0^{+}}^{q} T u\left(t_{2}\right)\right|$ tend to 0 . Consequently $T\left(B_{r}\right)$ is equicontinuous. From Arzela-Ascoli Theorem we deduce that $T$ is completely continuous operator.

Now we apply Leray-Schauder nonlinear alternative to prove that $T$ has at least one nontrivial solution in $E$. Letting $\Omega=\{u \in E:\|u\|<r\}$, for any $u \in \partial \Omega$, such that $u=\lambda T u, 0<\lambda<1$, we get, with the help of (39)

$$
|u(t)|=\lambda|T u(t)| \leq|T u(t)| \leq C_{1}(\psi(r)+\phi(r)+1) .
$$

Taking into account (43), we obtain

$$
\left|{ }^{c} D_{0^{+}}^{q} T u(t)\right| \leq \frac{C_{2}}{(1-\sigma) \Gamma(1-\sigma)}(\psi(r)+\phi(r)+1) .
$$

From (49), (50) and (34), we deduce that

$$
\|u\| \leq(\psi(r)+\phi(r)+1)\left(C_{1}+\frac{C_{2}}{(1-\sigma) \Gamma(1-\sigma)}\right)<r
$$

which contradicts the fact that $u \in \partial \Omega$. Lemma 3.5 allows us to conclude that the operator $T$ has a fixed point $u^{*} \in \Omega$ and then the fractional boundary value problem (1) has a nontrivial solution $u^{*} \in E$. The proof is completed.

\section{Existence of nonnegative solutions}

In this section we investigate the positivity of solution for the fractional boundary value problem (1). To do this, we show the following hypotheses.

$(H 1) f(t, u, v)=a(t) f_{1}(u, v)$, where $a \in C([0,1],(0, \infty))$ and $f_{1} \in C\left(\mathbb{R}_{+} \times \mathbb{R}, \mathbb{R}_{+}\right)$;

(H2) $0<\int_{0}^{1} \Psi(s) \frac{a(s)}{(1-s)^{3-q}} d s<\infty$, where

$$
\Psi(s)=\left\{\begin{array}{cc}
\alpha-\frac{(\eta-s)^{q-2}(1-s)^{3-q}}{(q-2)} & s \leq \eta, \\
\alpha & s>\eta .
\end{array}\right.
$$

Let us rewrite the function $u$ as

$$
u(t)=\frac{1}{\Gamma(q-2)} \int_{0}^{1} \frac{H(t, s)}{(1-s)^{3-q}} a(s) f_{1}\left(u(s),{ }^{c} D_{0^{+}}^{\sigma} u(s)\right) d s,
$$


where

$$
H(t, s)= \begin{cases}\frac{\left((t-s)^{q-1}(1-s)^{3-q}\right)}{(q-2)(q-1)}+t \alpha & \\ -\frac{t(\eta-s)^{q-2}(1-s)^{3-q}}{(q-2)}, & s \leq t, s \leq \eta, \\ t \alpha-\frac{t(\eta-s)^{q-2}(1-s)^{3-q}}{(q-2)}, & s>t, s \leq \eta, \\ \frac{\left((t-s)^{q-1}(1-s)^{3-q}\right)}{(q-2)(q-1)}+t \alpha, & s \leq t, s>\eta, \\ t \alpha, \quad & s>t, s>\eta .\end{cases}
$$

Then,

$$
{ }^{c} D_{0^{+}}^{\sigma} u(t)=\int_{0}^{1} \frac{H_{\sigma}(t, s)}{(1-s)^{3-q}} a(s) f_{1}\left(u(s),{ }^{c} D_{0^{+}}^{\sigma} u(s)\right) d s,
$$

where

$$
H_{\sigma}(t, s)=\left\{\begin{array}{l}
\frac{\left((t-s)^{(q-\sigma)-1}(1-s)^{3-q}\right)}{\Gamma(q-\sigma)}+\frac{\alpha t^{1-\sigma}}{\Gamma(\sigma) \Gamma(q-2)} \\
-\frac{t^{1-\sigma}(\eta-s)^{q-2}(1-s)^{3-q}}{\Gamma(\sigma) \Gamma(q-1)}, s \leq t, s \leq \eta, \\
\frac{\alpha t^{1-\sigma}}{\Gamma(\sigma) \Gamma(q-2)} \\
-\frac{t^{1-\sigma}(\eta-s)^{q-2}(1-s)^{3-q}}{\Gamma(\sigma) \Gamma(q-1)}, s>t, s \leq \eta, \\
\frac{\left((t-s)^{(q-\sigma)-1}(1-s)^{3-q}\right)}{\Gamma(q-\sigma)} \\
+\frac{\alpha t^{1-\sigma}}{\Gamma(\sigma) \Gamma(q-2)}, s \leq t, s>\eta, \\
\frac{\alpha t^{1-\sigma}}{\Gamma(\sigma) \Gamma(q-2)}, s>t, s>\eta .
\end{array}\right.
$$

Now we give the properties of the Green function $H(t, s)$.

Lemma 4.1. If $\alpha \geq \frac{1}{(q-2)}$, then $H(t, s)$ has the following properties

(i) $H(t, s), H_{\sigma}(t, s) \in C([0,1] \times[0,1]), 0<H(t, s), 0<H_{\sigma}(t, s)$ for all $t, s \in[0,1]$, 
(ii) if $t \in[\tau, 1], \tau>0$, then for all $s \in[0,1]$ we have

$$
0<\tau \Psi(s) \leq H(t, s) \leq 2 \Psi(s)
$$

and

$$
0<\frac{\tau}{\Gamma(\sigma) \Gamma(q-2)} \Psi(s) \leq H_{\sigma}(t, s) \leq \varsigma \Psi(s),
$$

where $\varsigma=\left(\frac{(q-2) \Gamma(\sigma)+1}{\Gamma(\sigma) \Gamma(q-2)}\right)$.

Proof. (i) It is obvious that $H(t, s) \in C([0,1] \times[0,1])$. Moreover, we have

$$
t \alpha-\frac{t(\eta-s)^{q-2}(1-s)^{3-q}}{(q-2)}=\frac{t\left[\alpha(q-2)-(1-s)^{3-q}(\eta-s)^{q-2}\right]}{(q-2)}
$$

which is positive if $\alpha \geq \frac{1}{(q-2)}$. Hence $H(t, s)$ is nonnegative for all $t, s \in$ $[0,1]$.

(ii) Let $t \in[\tau, 1]$. It is easy to see that $\Psi(s) \neq 0$. Then, we have

$$
\begin{gathered}
\frac{H(t, s)}{\Psi(s)}=\frac{\frac{\left((t-s)^{q-1}(1-s)^{3-q}\right)}{(q-2)(q-1)}+t \alpha}{\alpha} \\
\leq \frac{(1-s)^{2}}{(q-1)}+t \leq 2, \eta<s \leq t, \\
\times\left(\frac{H(t, s)}{\Psi(s)}=\frac{1}{\alpha-\frac{(\eta-s)^{q-2}(1-s)^{3-q}}{(q-2)}}\right. \\
\left.(t-s)^{q-1}(1-s)^{3-q}\right) \\
(q-2)(q-1) \\
=\frac{(t-s)^{q-1}(1-s)^{3-q}}{(q-1)\left(\alpha(q-2)-(\eta-s)^{q-2}(1-s)^{3-q}\right)}+t \\
\leq 2, \quad s \leq t, s \leq \eta,
\end{gathered}
$$




$$
\begin{gathered}
\frac{H(t, s)}{\Psi(s)}=\frac{\left[\frac{t \alpha}{(1-s)^{3-q}}-\frac{t(\eta-s)^{q-2}}{(q-2)}\right]}{\left[\frac{\alpha}{(1-s)^{3-q}}-\frac{(\eta-s)^{q-2}}{(q-2)}\right]} \\
=t \leq 2, \quad t<s \leq \eta, \\
\frac{H(t, s)}{\Psi(s)}=t \leq 2, \quad t<s, \eta<s .
\end{gathered}
$$

Now we look for lower bounds of $H(t, s)$ :

$$
\begin{aligned}
& \eta \leq s \leq t, \\
& \frac{H(t, s)}{\Psi(s)} \geq t \geq \tau, \quad s \leq t, s<\eta, \\
& t \leq s, s<\eta, \\
& t \leq s, \eta<s .
\end{aligned}
$$

Since $\Psi(s)$ is nonnegative, we obtain

$$
0<\tau \Psi(s) \leq H(t, s) \leq 2 \Psi(s)
$$

Similarly, we can prove that $H_{\sigma}(t, s)$ has the following properties. The proof is completed.

We recall the definition of positive solution.

Definition 4.2. A function $u$ is called positive solution of the fractional boundary value problem (1) if $\mathrm{u}(t) \geq 0$, for all $t \in[0,1]$.

Lemma 4.3. If $u \in E^{+}$and $\alpha \geq \frac{1}{(q-2)}$, then the solution of the fractional BVP (1) is nonnegative and satisfies

$$
\min _{t \in[\tau, 1]}\left(u(t)+{ }^{c} D_{0^{+}}^{\sigma} u(t)\right) \geq \frac{\tau(1+\Gamma(\sigma))}{1+q \Gamma(\sigma)}\|u\| .
$$

Proof. First, let us remark that under the assumptions on $u$ and $f$, the function ${ }^{c} D_{0^{+}}^{\sigma} u$ is nonnegative. Applying the right-hand side of inequality (54), we get

$$
u(t) \leq \frac{2}{\Gamma(q-2)} \int_{0}^{1} \Psi(s) \frac{a(s)}{(1-s)^{3-q}} f_{1}\left(u(s),{ }^{c} D_{0^{+}}^{\sigma} u(s)\right) d s .
$$


Moreover, (54) gives

$$
\begin{aligned}
{ }^{c} D_{0^{+}}^{\sigma} u(t) & =\int_{0}^{1} H_{\sigma}(t, s) \frac{a(s)}{(1-s)^{3-q}} f_{1}\left(u(s),{ }^{c} D_{0^{+}}^{\sigma} u(s)\right) d s \\
& \leq \varsigma \int_{0}^{1} \Psi(s) \frac{a(s)}{(1-s)^{3-q}} f_{1}\left(u(s),{ }^{c} D_{0^{+}}^{\sigma} u(s)\right) d s .
\end{aligned}
$$

Combining (63) and (64) yield

$$
\|u\| \leq\left(\frac{2}{\Gamma(q-2)}+\varsigma\right) \int_{0}^{1} \Psi(s) \frac{a(s)}{(1-s)^{3-q}} f_{1}\left(u(s),{ }^{c} D_{0^{+}}^{\sigma} u(s)\right) d s
$$

which is equivalent to

$$
\|u\| \leq\left(\frac{q \Gamma(\sigma)+1}{\Gamma(\sigma) \Gamma(q-2)}\right) \int_{0}^{1} \Psi(s) \frac{a(s)}{(1-s)^{3-q}} f_{1}\left(u(s),{ }^{c} D_{0^{+}}^{\sigma} u(s)\right) d s .
$$

Hence,

$$
\int_{0}^{1} \Psi(s) \frac{a(s)}{(1-s)^{3-q}} f_{1}\left(u(s),{ }^{c} D_{0^{+}}^{\sigma} u(s)\right) d s \geq \frac{\Gamma(\sigma) \Gamma(q-2)}{q \Gamma(\sigma)+1}\|u\| .
$$

In view of the left hand side of (54), we obtain for all $t \in[\tau, 1]$

$$
u(t) \geq \frac{\tau}{\Gamma(q-2)} \int_{0}^{1} \Psi(s) \frac{a(s)}{(1-s)^{3-q}} f_{1}\left(u(s),{ }^{c} D_{0^{+}}^{\sigma} u(s)\right) d s .
$$

On the other hand, we have

$$
{ }^{c} D_{0^{+}}^{\sigma} u(t) \geq \frac{\tau}{\Gamma(\sigma) \Gamma(q-2)} \int_{0}^{1} \Psi(s) \frac{a(s)}{(1-s)^{3-q}} f_{1}\left(u(s),{ }^{c} D_{0^{+}}^{\sigma} u(s)\right) d s .
$$

From (67) and (68), we get

$$
\begin{gathered}
\min _{t \in[\tau, 1]}\left(u(t)+{ }^{c} D_{0^{+}}^{\sigma} u(t)\right) \\
\geq \frac{\tau(1+\Gamma(\sigma))}{\Gamma(\sigma) \Gamma(q-2)} \int_{0}^{1} \Psi(s) \frac{a(s)}{(1-s)^{3-q}} f_{1}\left(u(s),{ }^{c} D_{0^{+}}^{\sigma} u(s)\right) d s
\end{gathered}
$$


and with the help of (66), we deduce to

$$
\min _{t \in(\tau, 1)}\left(u(t)+{ }^{c} D_{0^{+}}^{\sigma} u(t)\right) \geq \frac{\tau(1+\Gamma(\sigma))}{1+q \Gamma(\sigma)}\|u\| .
$$

This completes the proof.

Define the quantities $A_{0}$ and $A_{\infty}$ by

$$
A_{0}=\lim _{(|u|+|v|) \rightarrow o} \frac{f_{1}(u, v)}{|u|+|v|}, \quad A_{\infty}=\lim _{(|u|+|v|) \rightarrow \infty} \frac{f_{1}(u, v)}{|u|+|v|} .
$$

The case $A_{0}=0$ and $A_{\infty}=\infty$ is called superlinear case and the case $A_{0}=\infty$, $A_{\infty}=0$ is called sublinear case. The main result of this section is as follows.

Theorem 4.4. Under the assumptions of Lemma 4.3, the fractional BVP (1) has at least one nonnegative solution in the both cases superlinear as well as sublinear.

To prove Theorem 4.4, we apply the well-known Guo-Krasnosel'skii fixed point theorem on a cone.

Theorem 4.5. [15] Let $E$ be a Banach space and let $K \subset E$ be a cone. Assume that $\Omega_{1}$ and $\Omega_{2}$ are open subsets of $E$ with $0 \in \Omega_{1}, \bar{\Omega}_{1} \subset \Omega_{2}$ and let

$$
A: K \cap\left(\bar{\Omega}_{2} \backslash \Omega_{1}\right) \rightarrow K
$$

be a completely continuous operator such that

(i) $\|A u\| \leq\|u\|, u \in K \cap \partial \Omega_{1}$, and $\|A u\| \geq\|u\|, u \in K \cap \partial \Omega_{2}$,

(ii) $\|A u\| \geq\|u\|, u \in K \cap \partial \Omega_{1}$, and $\|A u\| \leq\|u\|, u \in K \cap \partial \Omega_{2}$.

Then, $A$ has a fixed point in $K \cap\left(\bar{\Omega}_{2} \backslash \Omega_{1}\right)$.

Proof. To prove Theorem 4.4, we define the cone $K$ by

$$
K=\left\{u \in E^{+}, \min _{t \in[\tau, 1]}\left(u(t)+{ }^{c} D_{0^{+}}^{\sigma} u(t)\right) \geq \frac{\tau(1+\Gamma(\sigma))}{1+q \Gamma(\sigma)}\|u\|\right\} .
$$

It is easy to check that $K$ is a nonempty closed and convex subset of $E$, hence it is a cone.

Using Lemma 4.3, we see that $T K \subset K$. From the proof of Theorem 3.4, we know that $T$ is completely continuous in $E$.

Let us prove the superlinear case. First, since $A_{0}=0$, for any $\varepsilon>0$, there exists $R_{1}>0$, such that

$$
f_{1}(u, v) \leq \varepsilon(|u|+|v|)
$$


for $0<|u|+|v| \leq R_{1}$, Letting $\Omega_{1}=\left\{u \in E,\|u\|<R_{1}\right\}$, for any $u \in K \cap \partial \Omega_{1}$, it yields

$$
\begin{aligned}
T u(t) & =\frac{1}{\Gamma(q-2)} \int_{0}^{1} H(t, s) \frac{a(s)}{(1-s)^{3-q}} f_{1}\left(u(s),{ }^{c} D_{0^{+}}^{\sigma} u(s)\right) d s \\
& \leq \frac{2 \varepsilon\|u\|}{\Gamma(q-2)} \int_{0}^{1} \Psi(s) \frac{a(s)}{(1-s)^{3-q}} d s .
\end{aligned}
$$

Moreover, we have

$$
\begin{aligned}
{ }^{c} D_{0^{+}}^{\sigma} T u(t) & \leq \varsigma \int_{0}^{1} \Psi(s) \frac{a(s)}{(1-s)^{3-q}} f_{1}\left(u(s),{ }^{c} D_{0^{+}}^{\sigma} u(s)\right) d s \\
& \leq \varsigma \varepsilon\|u\| \int_{0}^{1} \Psi(s) \frac{a(s)}{(1-s)^{3-q}} d s .
\end{aligned}
$$

From (75) and (76) we conclude

$$
\begin{gathered}
\|T u\| \leq\left(\frac{2}{\Gamma(q-2)}+\varsigma\right) \varepsilon\|u\| \int_{0}^{1} \Psi(s) \frac{a(s)}{(1-s)^{3-q}} d s, \\
\|T u\| \leq\left(\frac{q \Gamma(\sigma)+1}{\Gamma(\sigma) \Gamma(q-2)}\right) \varepsilon\|u\| \int_{0}^{1} \Psi(s) \frac{a(s)}{(1-s)^{3-q}} d s .
\end{gathered}
$$

In view of hypothesis $(\mathrm{H} 2)$, one can choose $\varepsilon$ such that

$$
\varepsilon \leq \frac{\Gamma(\sigma) \Gamma(q-2)}{q \Gamma(\sigma)+1} \frac{1}{\int_{0}^{1} \Psi(s) \frac{a(s)}{(1-s)^{3-q}} d s} .
$$

The inequalities (77) and (78) imply that $\|T u\| \leq\|u\|$, for all $u \in K \cap \partial \Omega_{1}$.

Second, in view of $A_{\infty}=\infty$, then for any $M>0$ there exists $R_{2}>0$, such that $f_{1}(u, v) \geq M(|u|+|v|)$ for $|u|+|v| \geq R_{2}$.

Let $R=\max \left\{2 R_{1},(1+q \Gamma(\sigma)) R_{2} / \tau(1+\Gamma(\sigma))\right\}$ and denote by $\Omega_{2}$ the open set $\{u \in E,\|u\|<R\}$. If $u \in K \cap \partial \Omega_{2}$ then

$$
\min _{t \in[\tau, 1]}\left(u(t)+{ }^{c} D_{0^{+}}^{\sigma} u(t)\right) \geq \frac{\tau(1+\Gamma(\sigma))}{1+q \Gamma(\sigma)}\|u\|=\frac{\tau(1+\Gamma(\sigma))}{1+q \Gamma(\sigma)} R \geq R_{2} .
$$


Using the left hand side of (54) and Lemma 4.3, we obtain

$$
\begin{aligned}
T u(t) & \geq \frac{\tau}{\Gamma(q-2)} \int_{0}^{1} \Psi(s) \frac{a(s)}{(1-s)^{3-q}} f_{1}\left(u(s),{ }^{c} D_{0^{+}}^{\sigma} u(s)\right) d s \\
& \geq \frac{\tau M\|u\|}{\Gamma(q-2)} \int_{0}^{1} \Psi(s) \frac{a(s)}{(1-s)^{3-q}} d s .
\end{aligned}
$$

Moreover, we get with the help of (68)

$$
\begin{aligned}
{ }^{c} D_{0^{+}}^{\sigma} T u(t) & \geq \frac{\tau}{\Gamma(\sigma) \Gamma(q-2)} \int_{0}^{1} \Psi(s) \frac{a(s)}{(1-s)^{3-q}} f_{1}\left(u(s),{ }^{c} D_{0^{+}}^{\sigma} u(s)\right) d s \\
& \geq \frac{\tau}{\Gamma(\sigma) \Gamma(q-2)} M\|u\| \int_{0}^{1} \Psi(s) \frac{a(s)}{(1-s)^{3-q}} d s .
\end{aligned}
$$

In view of (80) and (81) we can write

$$
T u(t)+{ }^{c} D_{0^{+}}^{\sigma} T u(t) \geq \frac{\tau(1+\Gamma(\sigma))}{\Gamma(\sigma) \Gamma(q-2)} M\|u\| \int_{0}^{1} \Psi(s) \frac{a(s)}{(1-s)^{3-q}} d s .
$$

Let us choose $M$ such that

$$
M \geq \frac{\Gamma(\sigma) \Gamma(q-2)}{\tau(1+\Gamma(\sigma)) \int_{0}^{1} \Psi(s) \frac{a(s)}{(1-s)^{3-q}} d s},
$$

then we get

$$
T u(t)+{ }^{c} D_{0^{+}}^{\sigma} T u(t) \geq\|u\| .
$$

Hence,

$$
\|T u\| \geq\|u\|, \forall u \in K \cap \partial \Omega_{2} .
$$

The first part of Theorem 4.5 implies that $T$ has a fixed point in $K \cap\left(\bar{\Omega}_{2} \backslash \Omega_{1}\right)$ such that $R_{2} \leq\|u\| \leq R$. To prove the sublinear case we apply similar techniques. The proof is complete.

In order to illustrate our results, we give the following examples.

Example 4.6. The fractional boundary value problem

$$
\begin{aligned}
{ }^{c} D_{0^{+}}^{7 \mid 3} u & =(\sin t)^{2} u+(1-t)^{3} D_{0^{+}}^{1 \mid 5} u+e^{t} \\
& u(0)=u^{\prime \prime}(0)=0, u^{\prime}\left(\frac{1}{3}\right)=\frac{1}{2} u^{\prime \prime}(1)
\end{aligned}
$$

has a unique solution in $E$. 
Proof. In this case we have $f(t, x, y)=(\sin t)^{2} x+(1-t)^{2} y+e^{t}$,

$2<q=\frac{5}{2}<3,0<\sigma=\frac{1}{5}<1, \alpha=\frac{1}{2}$ and

$$
|f(t, x, \bar{x})-f(t, y, \bar{y})| \leq(\sin t)^{2}|x-y|+(1-t)^{3}|\bar{x}-\bar{y}| .
$$

Then, $g(t)=(\sin t)^{2}, h(t)=(1-t)^{3}$ and some calculus gives

$$
\begin{array}{cl}
\left\|I_{0^{+}}^{q-1} g\right\|=0.15045, & I_{0^{+}}^{q-1} g(1)=0.14633, \\
I_{0^{+}}^{q-1} g\left(\frac{1}{3}\right)=3.6113 \times 10^{-3}, & I_{0^{+}}^{q-2} g(1)=0.46503, \\
A_{g}=0.38246, & C_{g}=0.38658, \\
\left\|I_{0^{+}}^{q-1} h\right\|=0.13677, & I_{0^{+}}^{q-1} h(1)=0.25075, \\
I_{0^{+}}^{q-1} h\left(\frac{1}{3}\right)=9.7076 \times 10^{-2}, & I_{0^{+}}^{q-2} h(1)=0.16120, \\
A_{h}=0.42843, & C_{h}=0.31445, \\
C_{g}+C_{h}=0.38658+0.31445=0.7043<1, \\
A_{g}+A_{h}=0.38246+0.42843=0.81089<\left(1-\frac{1}{5}\right) \Gamma\left(1-\frac{1}{5}\right) .
\end{array}
$$

Thus Theorem 3.2 implies that fractional boundary value problem (85) has a unique in $E$.

Example 4.7. The fractional boundary value problem

$$
\begin{gathered}
{ }^{c} D_{0^{+}}^{7 \mid 3}=(1-t)^{2}\left(\frac{u^{2}+2}{\left(6+u^{4}\right)}+\ln \left(1+\left({ }^{c} D_{0^{+}}^{\frac{5}{6}} u\right)^{2}\right)+1\right), \\
u(0)=u^{\prime \prime}(0)=0, u^{\prime}(\eta)=\frac{3}{2} u^{\prime \prime}(1)
\end{gathered}
$$

has at least one nontrivial solution in $E$.

Proof. We apply Theorem 3.4 to prove that the fractional boundary value problem (89) has at least one nontrivial solution. We have $q=\frac{7}{3}, \sigma=\frac{2}{3}, \alpha=\frac{3}{2}, \eta=\frac{1}{4}$ and

$$
\begin{aligned}
\mid f(t, x, \bar{x} \mid & =\left|\frac{(1-t)^{2}\left(x^{2}+2\right)}{\left(6+x^{4}\right)}+(1-t)^{2} \ln \left(1+\bar{x}^{2}\right)+(1-t)^{2}\right| \\
& \leq(1-t)^{2} \frac{\left(x^{2}+2\right)}{\left(6+x^{4}\right)}+(1-t)^{2} \ln \left(1+\bar{x}^{2}\right)+(1-t)^{2} \\
& \leq k(t) \psi(|x|)+h(t) \phi(|\bar{x}|)+g(t),
\end{aligned}
$$


where $k(t)=h(t)=g(t)=(1-t)^{2}, \psi(x)=\frac{\left(x^{2}+2\right)}{\left(6+x^{4}\right)}$,

$\phi(\bar{x})=\ln \left(1+\bar{x}^{2}\right), f(t, 0,0) \neq 0$.

Let us find $r$ such that (34) holds. For this, we have

$$
\begin{aligned}
\left\|I_{0^{+}}^{q-1} g\right\| & =0.25843, & I_{0^{+}}^{q-1} g(1) & =0.33595, \\
I_{0^{+}}^{q-1} g\left(\frac{1}{3}\right) & =0.14420, & I_{0^{+}}^{q-2} g(1) & =0.15998, \\
C_{g}=C_{1} & =0.50928, & & A_{g}=C_{2}=0.5868 .
\end{aligned}
$$

We see that (34) is equivalent to $\left(\left(\frac{r^{2}+2}{r^{4}+6}\right)+\ln \left(1+r^{2}\right)+1\right)(1.1664)-r$ which is negative for $r=6$.

Example 4.8. The fractional boundary value problem

$$
\begin{gathered}
{ }^{c} D_{0^{+}}^{q} u=\frac{1-t^{2}}{1+t^{2}}\left[\frac{4 \pi}{u+{ }^{c} D_{0^{+}}^{\sigma} u+6 \pi}+e^{-\pi\left(u+{ }^{c} D_{0^{+}}^{\sigma} u\right)}\right], \\
u(0)=u^{\prime \prime}(0)=0, u^{\prime}(\eta)=\alpha u^{\prime \prime}(1)
\end{gathered}
$$

has at least one nonnegative solution, if $q=\frac{7}{3}, \sigma=\frac{5}{6}, \alpha=\frac{7}{2}$ and $\eta=\frac{1}{4}$.

Proof.

$$
\begin{aligned}
& f\left(\left(t, u,{ }^{c} D_{0^{+}}^{\sigma} u\right)=\frac{1-t^{2}}{1+t^{2}}\left[\frac{4 \pi}{u+{ }^{c} D_{0^{+}}^{\sigma} u+6 \pi}+e^{-\pi\left(u+{ }^{c} D_{0^{+}}^{\sigma} u\right)}\right],\right. \\
& f_{1}\left(u,{ }^{c} D_{0^{+}}^{\sigma} u\right)=\left[\frac{4 \pi}{u+{ }^{c} D_{0^{+}}^{\sigma} u+6 \pi}+e^{-\pi\left(u+{ }^{c} D_{0^{+}}^{\sigma} u\right)}\right], \\
& a(t)=\frac{1-t^{2}}{1+t^{2}} .
\end{aligned}
$$

Obviously, it is not difficult to verify conditions (H1) and (H2). Through a simple calculation we get $A_{0}=0$ and $A_{\infty}=\infty$. Thus, by Theorem 4.4 we get that the problem (92) has at least one nonnegative solution.

\section{Bibliography}

[1] B. Ahmad and J. J. Nieto, Existence results for a coupled system of nonlinear fractional differential equations with three-point boundary conditions, Comput. Math. Appl. 58 (2009), 1838-1843. 
[2] B. Ahmad and J. J. Nieto, Existence of solutions for nonlocal boundary value problems of higher-order nonlinear fractional differential equations, Abstract and Applied Analysis, 2009 (2009), Article ID 494720, doi:10.1155/2009/494720.

[3] Z. Bai and H. Lu, Positive solutions for boundary value problem of nonlinear fractional differential equation, Journal of Mathematical Analysis and Applications 311(2) (2005), 495-505.

[4] K. Balachandran and J. J. Trujillo, The nonlocal Cauchy problem for nonlinear fractional integro differential equations in Banach spaces, Nonlinear Anal. 72 (2010), 4587-4593.

[5] M. Belmekki, J. J. Nieto and R. Rodriguez-Lopez, Existence of periodic solution for a nonlinear fractional differential equation, Bound. Value Probl. 2009 (2009), Article ID 324561.

[6] M. Benchohra, S. Hamani and S. K. Ntouyas, Boundary value problems for differential equations with fractional order and nonlocal conditions, Nonlinear Anal. 71 (2009), 2391-2396.

[7] Z. B. Bai, On positive solutions of a nonlocal fractional boundary value problem, Nonlinear Anal. 72 (2010), 916-924.

[8] Y. K. Chang and J. J. Nieto, Some new existence results for fractional differential inclusions with boundary conditions, Math. Comput. Model. 49 (2009), 605-609.

[9] Zhoujin Cui, Pinneng Yu and Zisen Mao, Existence of solutions for nonlocal boundary value problems of nonlinear fractional differential equations, Advances in Dynamical Systems and Applications 7(1) (2012), 31-40.

[10] K. Deimling, Nonlinear Functional Analysis, Springer, Berlin, Germany, 1985.

[11] M. El-Shahed, Positive solutions for boundary value problem of nonlinear fractional differential equation, Abstract and Applied Analysis 2007 (2007), Article ID 10368.

[12] A. Guezane-Lakoud and R. Khaldi, Solvability of a fractional boundary value problem with fractional integral condition, Nonlinear Analysis 75 (2012), 2692-2700.

[13] A. Guezane-Lakoud and R. Khaldi, Positive solution to a higher order fractional boundary value problem with fractional integral condition, Romanian Journal of Mathematics and Computer Sciences 2 (2012), 28-40.

[14] A. Guezane-Lakoud and R. Khaldi, Solvability of a three-point fractional nonlinear boundary value problem, Differ. Equ. Dyn. Syst. 20(4) (2012), 395-403.

[15] D. J. Guo and V. Lakshmikantham, Nonlinear Problems in Abstract Cones, Vol. 5 of Notes and Reports in Mathematics in Science and Engineering, Academic Press, USA, 1988.

[16] Ch. S. Goodrich, Existence of a positive solution to a class of fractional differential equations, Appl. Math. Lett. 23 (2010), 1050-1055. 
[17] R. Hilfer, Applications of Fractional Calculus in Physics, World Scientific, NJ, USA, 2000 .

[18] N. Heymans and I. Podlubny, Physical interpretation of initial conditions for fractional differential equations with Riemann-Liouville fractional derivatives, Rheologica Acta 37 (2005), 1-7.

[19] H. Jafari and V. Daftardar-Gejji, Positive solutions of nonlinear fractional boundary value problems using Adomian decomposition method, Applied Mathematics and Computation 180(2) (2006), 700-706.

[20] A. A. Kilbas, H. M. Srivastava, and J. J. Trujillo, Theory and Applications of Fractional Differential Equations, Vol. 204 of North-Holland Mathematics Studies, Elsevier, 2006.

[21] S. Liang and J. Zhang, Existence and uniqueness of positive solutions to m-point boundary value problem for nonlinear fractional differential equation, Journal of Applied Mathematics and Computing 38(1) (2012), 225-241.

[22] D. Mozyrska and D. F. M. Torres, Modified optimal energy and initial memory of fractional continuous-time linear systems, Signal Process 91 (2011), 379-385.

[23] K. B. Oldham, Fractional differential equations in electrochemistry, Advances in Engineering Software 41(1) (2010), 9-12.

[24] I. Podlubny, Fractional Differential Equations: An Introduction to Fractional Derivatives, Fractional Differential Equations, to Methods of Their Solution and Some of Their Applications, Vol. 198 of Mathematics in Science and Engineering, Academic Press, USA, 1998.

[25] T. Qiu and Z.Bai, Existence of positive solutions for singular fractional differential equations, Electronic Journal of Differential Equations, 2008 (2008), Article 146.

[26] M. Rehman and R. Ali, Positive solutions of nonlocal boundary value problem for higher order fractional differential system, Dynamic Systems and Applications 20 (2011), 169-182.

[27] S. G. Samko, A. A. Kilbas and O. I. Marichev, Fractional Integrals and Derivatives. Theory and Applications, Gordon and Breach, London, 1993.

[28] Y. Zhao, S. Sun, Z. Han and M. Zhang, Positive solutions for boundary value problems of nonlinear fractional differential equations, Applied Mathematics and Computation, 217(16) (2011), 6950-6958.

Received December 12, 2018; revised April 14, 2019; accepted May 4, 2019.

\section{Author information}

Assia Guezane-Lakoud, Department of Mathematics, Faculty of Science, University of

Badji Mokhtar, Annaba 23000, Algeria.

E-mail: a-guezane@yahoo.fr 
Kheireddine Belakroum, Department of Mathematics, Faculty of Exact Sciences, University of Mentouri Brothers-Constantine, Algeria.

E-mail: kheireddinebelakroum@gmail.com 